Portland State University

PDXScholar

4-2019

\title{
ADHD-Related Impairments in Error-Processing Predict Symptom Change
}

Amber Schwartz

Portland State University

Follow this and additional works at: https://pdxscholar.library.pdx.edu/honorstheses Let us know how access to this document benefits you.

\section{Recommended Citation}

Schwartz, Amber, "ADHD-Related Impairments in Error-Processing Predict Symptom Change" (2019). University Honors Theses. Paper 666.

https://doi.org/10.15760/honors.679

This Thesis is brought to you for free and open access. It has been accepted for inclusion in University Honors Theses by an authorized administrator of PDXScholar. Please contact us if we can make this document more accessible: pdxscholar@pdx.edu. 


\section{ADHD-Related Impairments in Error-Processing Predict Symptom Change}

By

Amber Schwartz

An undergraduate honors thesis submitted in partial fulfillment of the requirements for the degree of

Bachelor of Science

in

University Honors

and

Psychology

Thesis Adviser:

Sarah Karalunas, PhD.

Portland State University

2019 


\begin{abstract}
Attention-Deficit Hyperactivity Disorder (ADHD) is a common childhood disorder with heterogeneous symptom trajectories. Performance monitoring, which involves the ability to recognize errors and make behavioral adjustments, is one aspect of self-regulation that may contribute to symptom change. Early and late stages of error processing can be quantified via EEG-recorded event-related potentials. The final stage of performance monitoring, which involves making behavioral adjustments, is measured as post-error slowing. It is currently unclear whether impaired performance monitoring is associated with ADHD, specifically, or with comorbid symptoms of Oppositional Defiant Disorder (ODD). Here, better performance monitoring is hypothesized to be associated with ADHD, independent of comorbid ODD symptoms, and to predict ADHD symptom improvement. Method: 122 children from an ongoing longitudinal study $(\mathrm{Control}=60, \mathrm{ADHD}=62)$ completed behavioral ratings and semistructured clinical interviews to assess ADHD and comorbid ODD symptoms. At annual followup visits 5-8 years later, clinical assessment was repeated, and children completed a computerized emotional go/no-go with EEG recorded. Results: Controls had marginally larger $\operatorname{ERN}\left(p=.056, \eta^{2}=.03\right)$ and larger Pe amplitudes than ADHD participants $(F[1,118]=4.38$, $p=.04)$. Comorbid ODD did not explain these differences. Adolescents with ADHD also made less adaptive behavioral adjustments in emotional than in non-emotional conditions, $F(2,198)=3.758, p=.025$. Higher ERN amplitudes during positive conditions predicted greater ADHD symptom improvement, $\mathrm{R}^{2}=.057, p=.016$. Conclusions: Performance monitoring is associated with ADHD, independently of ODD. Emotional context does not affect error processing, but may interfere with adaptive behavioral adjustments for individuals with ADHD. Emotion dysregulation may also influence ADHD symptom trajectories.
\end{abstract}




\section{INTRODUCTION}

Attention-Deficit Hyperactivity Disorder is considered one of the most prevalent childhood disorders, affecting 5-6\% of children and adolescents (Michelini et. al, 2016), and an estimated 6.1 million children in the United States (Danielson et al., 2018). ADHD is typically diagnosed in the early or middle childhood period based on developmentally inappropriate levels of inattention, hyperactivity, and impulsivity (Shiels \& Hawk, 2010). The disorder is known to be highly heterogenous, which is demonstrated by differences in symptom presentation and cognitive impairment, as well as diverging developmental trajectories (Shiels \& Hawk, 2010). However, the reasons for these different trajectories are not clear. One possibility is that differences in children's ability to monitor and adjust their own behavior, also called performance monitoring, contributes to how much symptoms improve over time. Greater ability to monitor and adjust behavior may contribute to greater peer acceptance and academic achievement, as well as more improvements in ADHD symptoms. In addition, having more social support and reduced environmental stress could lead to healthier coping strategies (and less risky behavior), potentially lessening the impairing effects of ADHD symptoms across development. This research will examine differences in performance monitoring abilities using both behavioral and electroencephalogram measured event-related potentials (ERPs) to determine:1) if performance monitoring differences exists between ADHD and typicallydeveloping controls, 2) if any impairments in the ADHD group can be attributed to the presence of comorbid Oppositional Defiant Disorder, and 3) whether differences in performance monitoring help explain differences in ADHD symptom change over time. Results have implications for understanding the heterogeneous nature of ADHD and for improving clinical outcomes by determining which individuals need additional support early in childhood. 


\section{Performance monitoring in ADHD}

The ability to monitor performance, that is, to effectively recognize when mistakes are made and make behavioral adjustments, is critical for development, and particularly for developing adaptive behavior (Groen et al., 2008). There are several aspects of performance monitoring, which include error detection (with or without conscious awareness of the error), error awareness, and behavioral response adjustment. ERPs are one useful tool for measuring performance monitoring because distinct brain responses are related to each phase of error detection and error awareness. The two most common ERP components associated with performance monitoring are error-related negativity (ERN) and error-related positivity $(\mathrm{Pe})$, each of which is an electrophysiological neural response that occurs in response to the conflict generated by errors (Sur \& Sinha, 2009). The initial stage of performance monitoring is measured by examining ERN, which is a negative deflection occurring when the initial conflict between the correct and expected response is detected. The ERN is thought to be generated by the anterior cingulate cortex (ACC) (Shiels \& Hawk, 2010) and is present when an error is made, regardless of the individual's conscious awareness of the error (Groom et al., 2009). The ERN is typically followed by a positive deflection known as error-related positivity $(\mathrm{Pe})$ that is generally thought to reflect conscious error recognition (Groen et al., 2008).

There have been inconsistent findings regarding whether ERN is impaired in ADHD. Some studies find that children with ADHD have reduced ERN amplitudes when compared to children without ADHD (Groom et al., 2009; Meel et al., 2007; Marquardt et al., 2018; Liotti et al., 2005; Groen et al., 2008), but other studies fail to find any group differences (Zhang et al., 2009). There is more consistent evidence for impairments in error awareness (Pe) than error detection (ERN) in ADHD. Pe is generally thought to be impaired in ADHD (Shiels \& Hawk, 
2010), which is determined by smaller Pe amplitudes in ADHD groups (Jonkman et al., 2007;

Wiersema et al, 2009; Overtoom et al., 2002; Groen et al., 2008) Moreover, some studies have found differences in Pe even when there are not differences in ERN. For example, Wiersema et al. used a visual go/no-go computerized task on adults with and without ADHD to determine if error-processing deficits persisted into adulthood, and found that there were no differences in ERN, but that adults with ADHD displayed a smaller Pe (2009). Nonetheless, other studies find differences in both ERN and Pe (Groen et al., 2008). This was also true for Marquardt et al. (2018), who used a modified flanker task and found that overt behavioral responses were the same, but that the ADHD group had both reduced ERN and Pe amplitudes when compared to healthy controls. In part, these discrepancies may be related to differences in handling of comorbidity with the ADHD group. Other possibilities include small sample sizes, different ERP measuring techniques, the paradigms/tasks selected, and heterogeneity associated with ADHD. Overall, impairments in conscious error awareness are more consistently found in ADHD than impairments in error detection, but mixed findings suggest that more research is necessary to reach a consensus regarding the parts of performance monitoring that are impaired in ADHD.

ERPs are an important tool for understanding error detection, especially in the first two stages of performance monitoring, because error-processing it is an internal process that a person may not even be aware is happening. The last phase of performance monitoring, which involves adjusting behavior after making an error, can be measured using reaction times. In particular, post-error slowing is a behavioral response adjustment that can be measured on trials following an error (Shiels \& Hawk, 2010). It is usually determined by comparing the average reaction time following a correct response, to the reaction time following an incorrect response. Most typically-developing children are slower to respond after making a mistake, which helps them to 
be sure their next response is correct. Many studies have found that ADHD individuals make significantly more errors on cognitive tasks than non-ADHD controls, while showing less posterror slowing (Shalaine Payne, 2017; Wiersema et al., 2009; Jonkman et al., 2007; Schachar et al, 2004), suggesting that they exhibit poorer adaptive control (Shiels \& Hawk, 2010). This may be because children with ADHD are not recognizing their mistakes (which would be seen using ERN or Pe), or because even when they recognize mistakes, they are not able to change their behavior. By looking at all three phases of performance monitoring, this research will help clarify which parts of performance monitoring are affected in ADHD.

\section{Role of comorbid emotion dysregulation in ADHD}

The majority of studies on performance monitoring in ADHD have not accounted for comorbid externalizing disorders, such as Oppositional Defiant Disorder (ODD), which is characterized by argumentative behavior and severe anger or irritability. This is an important consideration because it is estimated that up to $35 \%$ of individuals diagnosed with ADHD also have ODD (Barkley, 2015). Unfortunately, the differences in error processing between ADHD and ODD groups, is not well understood or widely studied (Jarret \& Hilton, 2017). Some studies have suggested that externalizing behavior disorders (ADHD or ODD) are associated with reduced ERN amplitudes (Meyer \& Klein, 2018). However, it remains unclear whether impairments are due to attentional difficulties associated with ADHD, or more to comorbid ODD symptoms. In addition, there are even fewer studies that have examined whether Pe is related to comorbid ODD, and what effects ODD has on post-error slowing. Thus, the next question this research will attempt to answer is whether performance monitoring impairments in ADHD adolescents can be attributed to the presence of comorbid externalizing disorders. 
Even when children with ADHD do not meet full criteria for comorbid ODD, there is increasing evidence suggesting that emotion dysregulation is an important component of the disorder. Deficits in emotion regulation are associated with more emotional arousability, excitability, and lability, as well as more frustration, quickness to anger, impatience, and hostility (Barkley, 2015). Studies have found that some, but not all, children and adults with ADHD respond to emotional stimuli differently, with the majority of literature focusing on the dysregulation of negative emotions (Bunford et al., 2015). However, more research is also proposing that ADHD impairments exist for both the ability to regulate positive and negative emotions (Karalunas et al., 2019; Karalunas et al., 2014; Sjowall, 2012; Martel \& Nigg, 2006; Braaten \& Rosen, 2000). There is already evidence that individuals with ADHD may respond differently to emotional stimuli than typically-developing children. For example, by measuring reaction time on a visual-emotional oddball-paradigm, Raz \& Dan (2015) determined that ADHD participants responded slower to happy faces than controls, and responded the fastest to angry faces. This suggests that there might be stronger responses to negative stimuli, which is also consistent with the results of Shusjakova et al. (2018). Despite growing recognition of the importance of emotion in ADHD, studies have not yet examined how emotional context affects performance monitoring. Rather than using neutral tasks, this experimental study uses an emotional go/no-go with three emotion conditions in order to examine possible effects that emotion might have on performance monitoring.

\section{Relationships between performance monitoring and ADHD symptoms}

Lastly, this research will determine if performance monitoring ability can explain ADHD symptom changes across development. It is unclear why some individuals with ADHD appear to move into remission, while other see their ADHD symptoms persist into adulthood (Michelini et 
al., 2016). Perhaps the ability to monitor mistakes and adapt behaviors to avoid repeating those same mistakes is an important tool for learning and developing self-regulation. Therefore, impairments in conscious error-awareness, measured by diminished Pe amplitudes, may have important implications for development and symptom trajectories, with some studies finding diminished amplitudes associated with symptom persistence (Meel et al., 2007; Michelini et al., 2016). However, the first large scale study examining the relationship between performance monitoring and symptom change found that ADHD remitters had better overall error processing for both stages of performance monitoring (ERN and Pe) than ADHD persisters (Michelini et al, 2016). There is also little research examining post-error slowing and its potential relationship with symptom change. Overall, more longitudinal research is necessary to examine what stages of performance monitoring can potentially be associated with the remission or persistence of ADHD symptoms.

\section{Current Study}

The current study examines whether differences in performance monitoring on an emotional go/no-go task are related directly to ADHD or are explained by the comorbid presence of Oppositional Defiant Disorder. For the first time in the literature, we also consider whether these impairments change under different emotional conditions. Finally, the study examines whether differences in performance monitoring can help explain heterogeneity in symptom trajectories in ADHD. We hypothesize that 1) ADHD participants will be more impaired on measures of performance monitoring (ERN, Pe, post-error slowing) when compared to controls;

2) ADHD participants with and without comorbid ODD will have equally impaired performance monitoring (indicating that both disorders have impairments in error processing); and 3) that better performance monitoring will predict greater ADHD symptom remission. 


\section{METHODS}

\section{Participants}

Methods are also described in Alperin et al. (2017). 122 participants were recruited from a larger ongoing longitudinal study to participate in an additional EEG visit onsite. Although the larger study was longitudinal, each child participated in a single EEG visit. The EEG visit occurred at either Year 5, 6, or 8 of the longitudinal study depending on when the child enrolled. Participants were initially recruited from the Portland, OR area using community-based strategies such as public advertising and local outreach. This study was approved by the Institutional Review Boards at Oregon Health and Science University. Participants recruited from the longitudinal study were between 7-11 years of age when they enrolled and therefore 1216 years old at follow up.

Individuals were initially screened over the phone, and upon admittance into the study, parents/legal guardians we given and asked to sign written informed consents and adolescents written assent. Parents/legal guardians and teachers were then asked to complete a series of standardized assessments including the ADHD Rating Scale (ADHD-RS V) and Connors Rating Scale, $3^{\text {rd }}$ edition. Parents also completed an onsite semi-structured clinical interview (Kiddie Schedule for Affective Disorders and Schizophrenia, K-SADS) administered by a clinician, where they reported both current and lifetime symptom levels and impairment, as well as age of onset. Other assessments were also administered to adolescents, including a two-subset short form of WISC-IV (Block Design and Vocabulary) to measure IQ.

Symptom counts for inattention and hyperactivity-impulsivity were made using the parent (KSAD) and teacher (ADHD-RS) scores. These scores were combined using an "Or" 
algorithm. If either the parent or the teacher endorsed a symptom then it was counted. To be in the ADHD group, children needed to have at least 6 symptoms in at least one of the symptom domains (inattention or hyperactivity-impulsivity). To be in the control group, children needed to have 4 or fewer current ADHD symptoms, 3 or fewer inattentive symptoms, and 3 or fewer hyperactive symptoms. Children also needed to meet DSM criteria for cross-situational severity and impairment on the KSAD.

\section{Exclusion Criteria at Baseline}

Participants were screened over the phone for medication usage and neurological impairments. They were excluded if they were prescribed long-acting, non-stimulant psychotropic medications, had a history of seizures or head injuries that lead to loss of consciousness, had a history of substance use, were previously diagnosed with an intellectual disability, autism spectrum disorder, or psychosis. Individuals experiencing major depressive episodes and those with IQ<70 were also excluded from participating.

\section{Medication Washout}

All adolescents with ADHD who were taking stimulant medications were required to be off their stimulant medications for either 24 hours (short-lasting stimulants) or 48 hours (longlasting stimulants) prior to the EEG testing.

\section{ODD Ratings}

Oppositional Defiant Disorder symptoms were assessed using the symptom count from the KSAD. On this measure, each DSM symptom is rated as "present (1)" "subthreshold (0.5)," or "absent (0)". The symptom count is the sum of each symptom rating. For the analyses, participants with ADHD were assigned to either an ADHD-Only Group (ODD symptoms < 2.5, 
$\mathrm{n}=48$ ), an ADHD-ODD Group (ODD symptoms > 2.5, $\mathrm{n}=12$ ), or excluded (ODD symptoms $=2.5, \mathrm{n}=2$ ). The ADHD-Only and ADHD-ODD groups were compared to each other and to the Control group.

\section{Symptom Change}

ADHD symptom change was determined by subtracting the total number of ADHD symptoms (combined OR algorithm KSAD + teacher ADHD-RS as described above) at year one of the longitudinal study from the current number of ADHD symptoms assessed at follow up EEG testing visit.

\section{Experimental Procedure}

The emotional go/no-go task consisted of three separate conditions (happy, neutral, fear) that were counterbalanced, with $70 \%$ go trials and $30 \%$ no-go trials. For each condition, participants were shown a series of 12 grey scaled faces (50\% male, $50 \%$ female) from the NimStim set (Tottenham et al., 2009) and asked to respond to specific targets by pressing the spacebar on the computer keyboard. A total of 172 trials were presented for each condition, divided into two equal blocks (86 trials each). Condition order was counterbalanced across participants. In the happy condition, participants were instructed to respond via a key press to all happy faces, and to withhold a response for neutral faces. Likewise, in the fear condition, participants responded only to the fear faces and inhibited responses to neutral faces. The neutral condition consisted of all neutral faces in which participants were instructed to only respond to either the male faces or female faces, depending on the block (which was counterbalanced). Prior to testing, participants were given a short practice task to ensure understanding. 


\section{Post-error slowing}

Post-error slowing was measured on correct go trials following no-go errors (i.e., after the participant responded to a face when they were supposed to inhibit). Participants who did not have any no-go errors followed by a correct go trial were excluded from analysis, so the final sample size for this analysis was $\mathrm{ADHD}=57, \mathrm{Control}=46$. Post-error slowing was calculated as the difference between the average RT for correct Go trials not preceded by errors and the RT for correct Go trial immediately following errors.

\section{ERP Recordings and Analysis}

EEG recording and data preprocessing were identical to Alperin et al. (2017). EEG was recorded at $500 \mathrm{~Hz}$ with $32 \mathrm{Ag}-\mathrm{AgCl}$ active electrodes using PyCorder v1.0.9. The electrode array was based on the international 10-20 system centered at Cz. EEG signals were amplified by a BrainVision actiCHamp2 amplifier (Cary, NC). EEG data were analyzed using ERPLAB and EEGLAB toolboxes for MATLAB. Steps included: resampling to $250 \mathrm{~Hz}$, re-referencing to the average of all electrodes; filtering using an IIR filter with a bandwidth of .01-50 Hz; and removing eye artifacts independent component analysis. Recordings were referenced to $\mathrm{Cz}$ online then re-referenced offline. Response-locked epochs were created by time-locking to each incorrect no-go response. A baseline period of $-600,-400 \mathrm{~ms}$ was used corresponding to approximately -200 to $0 \mathrm{~ms}$ before the response. Trials not used in the analyses if they contained baseline drift or movement artifacts greater than $90 \mu \mathrm{V}$. Based on a priori criteria, a participant's data for a given condition were excluded from analyses if $>50 \%$ of the total trials were rejected due to artifacts. Pe and ERN amplitudes were measured using numerical integration at site $\mathrm{Cz}$ (See Figure 1), which is a common site for analyzing error-awareness components during 
adolescence. Pe was measured between latencies 200 and 400ms following an incorrect no-go trial. ERN was measured between -100 and 100ms also using numerical integration.

\section{Data Analyses}

All data was analyzed using SPSS v 24. Analysis included a series of repeated measures ANOVAs, both with and without covariates (age and sex), as well as a linear regression.

\section{RESULTS}

\section{Sample description}

Adolescents with ( $\mathrm{M}=13.7$ years) and without ADHD (M=14.0 years) did not significantly differ in age ( $p=.232)$, but those with ADHD did have lower IQ scores $(p=.003)$. The ADHD group also had more males $(75.8 \%)$ than females $(24.2 \%)$. However, when compared to the proportion of males in the control group (60\%), there were no significant differences in sex ( $p=.069)$. See Table 1 for a description of the sample.

\section{Group Differences}

ERN and Pe Amplitudes. There were 122 participants (ADHD=62, Control=60). 2 (group: ADHD, Control) x 3 (emotion: happy, neutral, fear) repeated measures ANOVAs were run with sex and age as covariates. There was a marginally significant difference between the ADHD group $(\mathrm{M}=1.040, \mathrm{SD}=0.072)$ and Control group $(\mathrm{M}=1.240, \mathrm{SD}=0.073)$ on mean $\mathrm{ERN}$ amplitudes, $F(1,118)=3.719, p=.056, \eta^{2}=.03$. The ADHD group trended towards having smaller ERNs. There were no main effects of Emotion $F(2,236)=1.808, p=.166$, and no significant ADHD x Emotion interaction $F(2,236)=.412, p=.663$. See Figure 2 and Figure 3. 
A separate 2 (group: ADHD, Control) x 3 (emotion: happy, neutral, fear) repeated measures ANOVA showed there was a significant relationship between ADHD status and Pe amplitudes, with the Control group $(\mathrm{M}=1.728, \mathrm{SD}=0.121)$ having higher mean Pe amplitudes than the ADHD group $(\mathrm{M}=1.369, \mathrm{SD}=0.119), F(1,118)=4.384, p=.038, \eta^{2}=.036$. There were no main effects of emotion, $F(2,236)=1.321, p=.269$, and no significant interaction, $F(2,236)=.681$, $p=.507$. See Figure 2 and Figure 3.

Post-Error Slowing. A 2 (group: ADHD, Control) x 3 (emotion: happy, neutral, fear) repeated measures ANOVAs showed there were no significant post-error slowing differences between groups $F(1,99)=.033, p=.86$, and no main effect of emotion, $F(2,198)=.776, p=.46$. However, there was a significant interaction between emotion and ADHD status, $F(2,198)=3.758, p=.025, \eta^{2}=.04$. In other words, the ADHD group had a faster response time following errors in the Happy condition than controls, but a slower response time following errors in the Fear condition than controls. They did not differ from controls in the neutral condition. See Figure 4.

\section{Comorbidity Group Differences}

ERN and Pe Amplitudes. A 3 (group: ADHD-only, ADHD-ODD, Control) x 3 (emotion: happy, neutral, fear) repeated measures ANOVA showed there was no significant difference in ERN amplitudes between the three symptom groups, $F(2,115)=2.385, p=.097, \eta^{2}=.040$. There were also no main effects of emotion and no interaction.

A separate 3 (group: ADHD-only, ADHD-ODD, Control) x 3 (emotion: happy, neutral, fear) repeated measures ANOVA showed there was a marginally significant difference in Pe amplitudes between the symptom groups, $F(2,115)=2.989, p=.054$. Controls had larger Pe 
amplitudes than both the ADHD-Only and ADHD/ODD groups across all conditions. The ADHD-only group had a trend towards higher Pe amplitudes than the ADHD/ODD group.

\section{Predicting Symptom Change}

There was no significant relationship between ERN Fear or Gender and symptom change (all $p>.49$ ). Adding covariates to the model did not change the results. However, larger Happy ERN predicted greater symptom improvement $\left(\mathrm{R}^{2}=.057, p=.016\right)$. When covariates were added to the model, ERN remained marginally significant $\left(\mathrm{R}^{2}=.105, R^{2} \Delta=.031, p=.070\right)$.

There were no significant relationships between Pe and symptom change (all $p>.38$ ). Adding covariates to the model did not change the results.

There were no significant relationships between post-error slowing and symptom change (all $p>.37$ ). Adding covariates to the model did not change the results.

\section{DISCUSSION}

This research examined if performance monitoring abilities are different between ADHD adolescents and controls. The second aim of this research was to determine if performance monitoring impairments were primarily attributed to the comorbid presence of Oppositional Defiant Disorder. Lastly, we examined if performance monitoring components could predict ADHD symptom trajectories.

Results showed group differences for both components of performance monitoring. The ADHD group appeared to have slightly smaller ERN amplitudes than controls, suggesting that initial error detection and general monitoring may be slightly more impaired in adolescents with ADHD. This aligns with the research of Groom et al. (2009) and Meel et al. (2007), who also 
reported diminished ERN amplitudes in the ADHD group compared to controls. These results differ from other studies, which found no group differences for ERN (Wiersema et al., 2009; Zhang et al., 2009). The ADHD group also appeared to have smaller Pe amplitudes than controls, which is consistent with other studies (Marquardt et al., 2018; Wiersema et al., 2009), and suggest a greater lack in conscious error awareness. Our research supports prior research that has found more consistent evidence for impairments in Pe than ERN (Shiels \& Hawk, 2010). The group differences found in this study were consistent across all of the emotion conditions, so neurophysiological evidence did not support any specific effects of emotion on error processing.

Overall, results suggest that error processing is impaired in ADHD adolescents and that the ability to detect errors is not dependent on emotion. In contrast, post-error slowing differences in ADHD adolescents did appear to be dependent on emotion. The ability to make behavioral adjustments following a mistake was impaired for both fear and happy emotions but in opposite ways. In the happy condition, ADHD adolescents exhibited less post-error slowing, and therefore impaired behavioral adjustment, compared to controls. On the other hand, ADHD adolescents showed more post-error slowing than controls on the fear emotion conditions. This demonstrates the importance of considering emotion regulation differences in ADHD adolescents. Literature suggests that individuals with ADHD may have difficulty regulating emotions and therefore show a stronger response to both positive and negative emotional stimuli. In this case, adolescents with ADHD may get overly excited and display a greater approach response to positive stimuli (the happy faces), but a greater withdrawal response to negative stimuli (fear faces), both of which prevent them from appropriately adjusting their behavior. Overall, results suggest that adolescents with ADHD have impaired error processing that does 
not vary by emotion, but more difficulty adjusting behavior in emotional than non-emotional conditions.

When examining the three ODD-related symptom groups, the ERN amplitudes for ADHD adolescents with and without ODD symptoms and the controls were not significantly different, suggesting that initial unconscious error awareness and monitoring ability was about the same. In other words, ODD did not appear to have a significant effect on unconscious errorawareness for ADHD individuals. However, results showed marginally significant differences for Pe amplitudes between the three groups, with the ADHD-ODD having marginally smaller mean Pe amplitudes than the ADHD-Only group. This could possibly indicate that ODD symptoms may have an influence on conscious error-awareness, although the effect may be small.

ADHD total symptom change was only predicted by ERN amplitudes for the happy condition. Higher ERN amplitude was associated with greater symptom improvement, and thus a greater decrease in the total number of ADHD symptoms. Michelini et al. (2016) also found that higher ERN amplitudes were associated with ADHD remitters, but that remitters also had higher Pe amplitudes. Our study showed that Pe was not associated with symptom change. The ability to regulate behavior and learn from mistakes in a variety of situations throughout adolescence may result in less overall ADHD symptom impairment. Therefore, better error detection in childhood could result in improved attention in late adolescence. This has clinical significance, because more interventions and additional assistance could be recommended for those individuals determined to be more at risk for symptom persistence.

While initial unconscious error-awareness had some ADHD predictability, conscious error-awareness was not predictive of ADHD symptom change, which was opposite of what was 
hypothesized. Therefore, our results are more closely aligned with Michelini et al. (2016), who found that ADHD remitters had higher ERN amplitudes. Post-error slowing was also not predictive of symptom change.

\section{Limitations}

Findings should be interpreted considering several limitations. First, only one of our measures of performance monitoring predicted symptom change. Replicating the effect will be important for making sure it is not a false positive finding. Nonetheless, the ability to predict future symptom trajectories from a single measure of error-awareness earlier in childhood should not be taken lightly. The complexity of ADHD and the fact that this study was able to predict change over the course of 5-8 years provides more evidence for the importance of performance monitoring ability and emotion regulation in childhood. With earlier identification of ADHD children at risk for symptom persistence, hopefully additional help and resources will be made available. Additional help may come in many forms, including additional therapy/counseling for emotional and behavioral struggles, participation in afterschool learning programs, one on one tutoring, additional academic support, and individualized school curricula.

Another limitation to this study was that we had a very small sample of ADHD participants with comorbid ODD $(n=12)$. This may mean the lack of statistical power necessary to capture the real group differences if they existed. The reason for the lack of comorbid ODD is because children with comorbid ODD were not specifically targeted when our participants were recruited. Additional studies with larger comorbid groups are needed.

Lastly, it would have been more effective if our study design had performance monitoring data from year 1, instead of only at follow up. Although recording EEG measures 
years after the participants were initially recruited is not ideal, this method of data collection is not uncommon. This approach of collecting ERP data at later time points is similar to what other longitudinal studies have also done. The reason for our research design is partly because the EEG testing was not part of the initial longitudinal design. Even without year 1 EEG data, we were still able to have some confidence in predicting ADHD symptom change partly due to the assumption of rank order stability across development. The ERN and Pe measures are considered biomarkers (Martin et al., 2018), and we can generally assume that an individual's overall rank on these performance monitoring measures will remain relatively consistent across time.

\section{Conclusions}

The current study found that children with ADHD have impairments in error detection and awareness that do not depend on emotion, but that problems adjusting behavior are worse under emotional than non-emotional conditions. In addition, impairments were generally not attributable to comorbid ODD. Finally, differences in error detection, particularly in positive emotion conditions, predicted ADHD symptom change. These results contribute to understanding how performance monitoring relates to symptom change, and to understanding emotion regulatory processes in ADHD. 


\section{REFERENCES}

Alperin, B. R., Gustafsson, H., Smith, C., \& Karalunas, S. L. (2017). The relationship between early and late event-related potentials and temperament in adolescents with and without ADHD. PloS one, 12(7), e0180627. doi:10.1371/journal.pone.0180627

Barkley, R. A. (2015). Emotional dysregulation is a core component of ADHD. In AttentionDeficit Hyperactivity Disorder: A Handbook for Diagnosis and Treatment (4th ed., pp. 81-115). New York, NY: The Guilford Press.

Braaten, E. B., \& Rosén, L. A. (2000). Self-regulation of affect in attention deficit-hyperactivity disorder (ADHD) and non-ADHD boys: Differences in empathic responding. Journal of Consulting and Clinical Psychology, 68(2), 313-321. http://dx.doi.org/10.1037/0022006X.68.2.313

Bunford, N., Evans, S. W., \& Wymbs, F. (2015). ADHD and emotion dysregulation among children and adolescents. Clinical Child and Family Psychology Review, 18(3), 185-217. doi:10.1007/s10567-015-0187-5

Danielson, M. L., Bitsko, R. H., Ghandour, R. M., Holbrook, J. R., Kogan, M. D., \& Blumberg, S. J. (2018). Prevalence of parent-reported ADHD diagnosis and associated treatment among U.S. children and adolescents, 2016. Journal of Clinical Child \& Adolescent Psychology, 47(2), 199-212. doi:10.1080/15374416.2017.1417860

Groen, Y., Wijers, A. A., Mulder, L. J., Waggeveld, B., Minderaa, R. B., \& Althaus, M. (2008). Error and feedback processing in children with ADHD and children with autistic spectrum disorder: An EEG event-related potential study. Clinical Neurophysiology, 119(11), 2476-2493. doi:10.1016/j.clinph.2008.08.004 
Groom, M. J., Cahill, J. D., Bates, A. T., Jackson, G. M., Calton, T. G., Liddle, P. F., \& Hollis, C. (2009). Electrophysiological indices of abnormal error-processing in adolescents with attention deficit hyperactivity disorder (ADHD). Journal of Child Psychology and Psychiatry,51(1), 66-76. doi:10.1111/j.1469-7610.2009.02128.x

Jarrett, M. A. and Hilton, D. C. (2017). Cognitive functions. In The Wiley Handbook of Disruptive and Impulse-Control Disorders. Eds J. E. Lochman and W. Matthys, pp. 159174). doi:10.1002/9781119092254.ch10

Jonkman, L., Melis, J. J., Kemner, C., \& Markus, C. R. (2007). Methylphenidate improves deficient error evaluation in children with ADHD: An event-related brain potential study. Biological Psychology, 76(3), 217-229. doi:10.1016/j.biopsycho.2007.08.004

Karalunas, S. L., Fair, D., Musser, E. D., Aykes, K., Iyer, S. P., \& Nigg, J. T. (2014). Subtyping attention-deficit/hyperactivity disorder using temperament dimensions: Toward biologically based nosologic criteria. JAMA psychiatry, 71(9), 1015-1024.

Karalunas, S. L., Gustafsson, H. C., Fair, D., Musser, E. D., \& Nigg, J. T. (2019). Do we need an irritable subtype of ADHD? Replication and extension of a promising temperament profile approach to ADHD subtyping. Psychological Assessment, 31(2), 236.

Liotti, M., Pliszka, S. R., Perez, R., Kothmann, D., \& Woldorff, M. G. (2005). Abnormal brain activity related to performance monitoring and error detection in Children with ADHD. Cortex,41(3), 377-388. doi:10.1016/s0010-9452(08)70274-0

Martel, M. M., \& Nigg, J. T. (2006). Child ADHD and personality/temperament traits of reactive and effortful control, resiliency, and emotionality. Journal of Child Psychology and Psychiatry, 47(11), 1175-1183. doi:10.1111/j.1469-7610.2006.01629.x 
Martin, E. A., Mccleery, A., Moore, M. M., Wynn, J. K., Green, M. F., \& Horan, W. P. (2018). ERP indices of performance monitoring and feedback processing in psychosis: A metaanalysis. International Journal of Psychophysiology, 132, 365-378. doi:10.1016/j.ijpsycho.2018.08.004

Marquardt, L., Eichele, H., Lundervold, A. J., Haavik, J., \& Eichele, T. (2018). Event-relatedpotential (ERP) correlates of performance monitoring in adults with attention-deficit hyperactivity disorder (ADHD). Frontiers in psychology, 9, 485.

doi:10.3389/fpsyg.2018.00485

Meel, C. S., Heslenfeld, D. J., Oosterlaan, J., \& Sergeant, J. A. (2007). Adaptive control deficits in attention-deficit/hyperactivity disorder (ADHD): The role of error processing. Psychiatry Research, 151(3), 211-220. doi:10.1016/j.psychres.

Meyer, A., \& Klein, D. N. (2018). Examining the relationships between error-related brain activity (the ERN) and anxiety disorders versus externalizing disorders in young children: Focusing on cognitive control, fear, and shyness. Comprehensive Psychiatry, 87, 112119. doi:10.1016/j.comppsych.2018.09.009

Michelini, G., Kitsune, G. L., Cheung, C. H., Brandeis, D., Banaschewski, T., Asherson, P., Kuntsi, J. (2016). Attention-deficit/hyperactivity disorder remission is linked to better neurophysiological error detection and attention-vigilance processes. Biological Psychiatry, 80(12), 923-932. doi:10.1016/j.biopsych.

Overtoom, C. C., Kenemans, J., Verbaten, M. N., Kemner, C., Molen, M. W., Engeland, H. V.,... Koelega, H. S. (2002). Inhibition in children with attention-deficit/hyperactivity 
disorder: A psychophysiological study of the stop task. Biological Psychiatry, 51(8), 668676. doi:10.1016/s0006-3223(01)01290-2

Payne, S. (2017). Error processing in attention deficit hyperactivity disorder. ProQuest Information and Learning, 78, 1-18

Raz, S., \& Dan, O. (2015) Altered event-related potentials in adults with ADHD during emotional faces processing. Clinical Neurophysiology. 126:514-523.

Schachar, R.J., Chen, S., Logan, G.D., Ornstein, T.J., Crosbie, J., Ickowicz, A., Pakulak, A. (2004). Evidence for an error monitoring deficit in attention deficit hyperactivity disorder. Journal of Abnormal Child Psychology, 32: 285. https://doi.org/10.1023/B:JACP.0000026142.11217.f2

Shiels, K., \& Hawk, L. W. (2010). Self-regulation in ADHD: The role of error processing. Clinical Psychology Review, 30(8), 951-961. doi:10.1016/j.cpr.

Shushakova, A., Ohrmann, P., \& Pedersen, A. (2018). Exploring deficient emotion regulation in adult ADHD: Electrophysiological evidence. European Archives of Psychiatry and Clinical Neuroscience, 268(4), 359-371. doi:10.1007/s00406-017-0826-6

Sjöwall, D., Roth, L., Lindqvist, S., \& Thorell, L. B. (2012). Multiple deficits in ADHD: Executive dysfunction, delay aversion, reaction time variability, and emotional deficits. Journal of Child Psychology and Psychiatry, 54(6), 619-627. doi:10.1111/jcpp. 12006

Sur, S., \& Sinha, V. K. (2009). Event-related potential: An overview. Industrial psychiatry journal, 18(1), 70-3. 
Tottenham, N., Tanaka, J. W., Leon, A. C., Mccarry, T., Nurse, M., Hare, T. A., Marcus, D. J., Westerlund, A., Casey, BJ., Nelson, C. (2009). The NimStim set of facial expressions: Judgments from untrained research participants. Psychiatry Research, 168(3), 242-249. doi:10.1016/j.psychres.2008.05.006

Wiersema, J.R., van der Meere, J.J. \& Roeyers, H. J. (2009). ERP correlates of error monitoring in adult ADHD. Neural Transmission, 116: 371. https://doi.org/10.1007/s00702-0080165-X

Zhang, J., Wang, Y., Cai, R., \& Yan, C. (2009). The brain regulation mechanism of error monitoring in impulsive children with ADHD — An analysis of error related potentials. Neuroscience Letters,460(1), 11-15. doi:10.1016/j.neulet.2009.05.027 
Table 1. Sample Description, $n=122(\mathrm{ADHD}=62$, Control=60).

\begin{tabular}{|l|l|l|l|}
\hline \multicolumn{1}{|c|}{ Variable } & \multicolumn{1}{|c|}{ Control } & \multicolumn{1}{c|}{ ADHD } & \multicolumn{1}{c|}{ Statistics } \\
\hline Age (years) & $14.0(1.1)$ & $13.7(1.5)$ & $\mathrm{p}=.232(\mathrm{~ns})$ \\
\hline Sex (\% male) & $60 \%$ & $75.8 \%$ & $\mathrm{p}=.069$ (ns) \\
\hline IQ & $116.3(12.1)$ & $106.9(13.9)$ & $\mathrm{p}=.003$ \\
\hline$\%$ White, non-Hispanic & $75.40 \%$ & $75.40 \%$ & $\mathrm{p}=.995(\mathrm{~ns})$ \\
\hline Stimulant Medication & N/A & $61.80 \%$ & $\mathrm{~N} / \mathrm{A}$ \\
\hline Median Income & $75-100 \mathrm{~K}$ & $35-50 \mathrm{~K}$ & $\mathrm{p}=.021$ \\
\hline
\end{tabular}


Figure 1: Headplot featuring site of 32 channel electroencephalogram recording at electrode Cz.

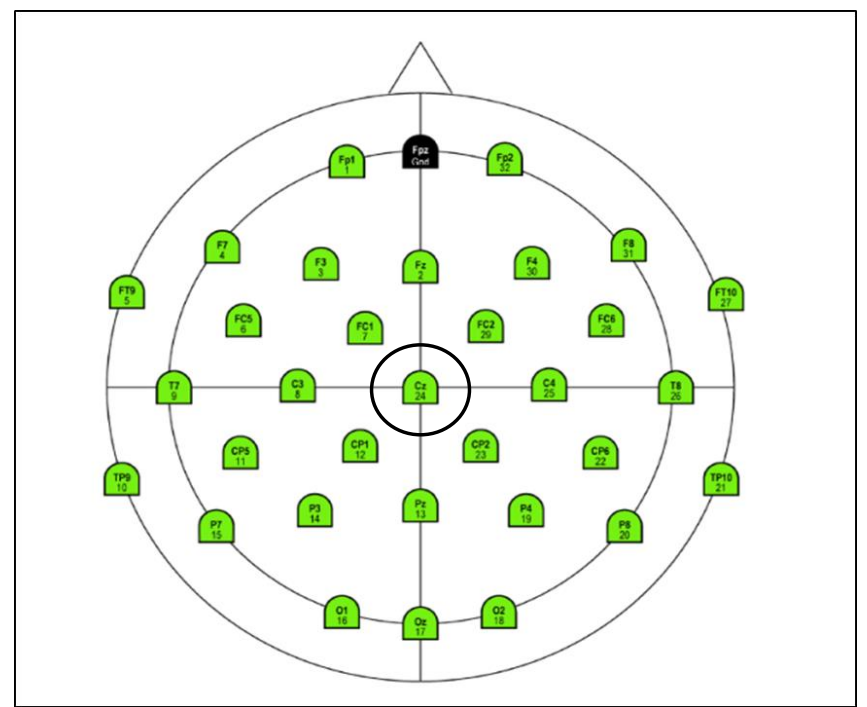


Figure 2. ADHD response-locked ERPs across emotion conditions.

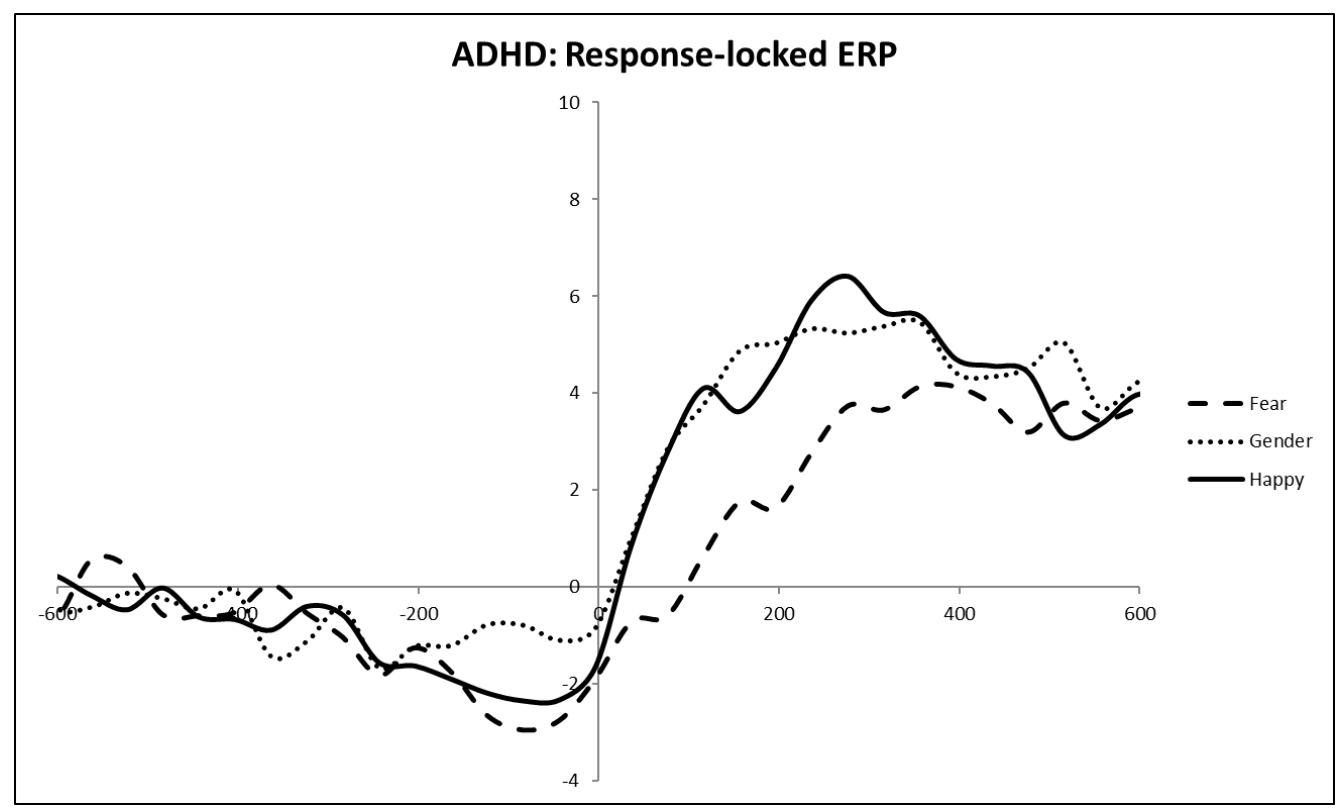


Figure 3. Control response-locked ERPs across emotion conditions.

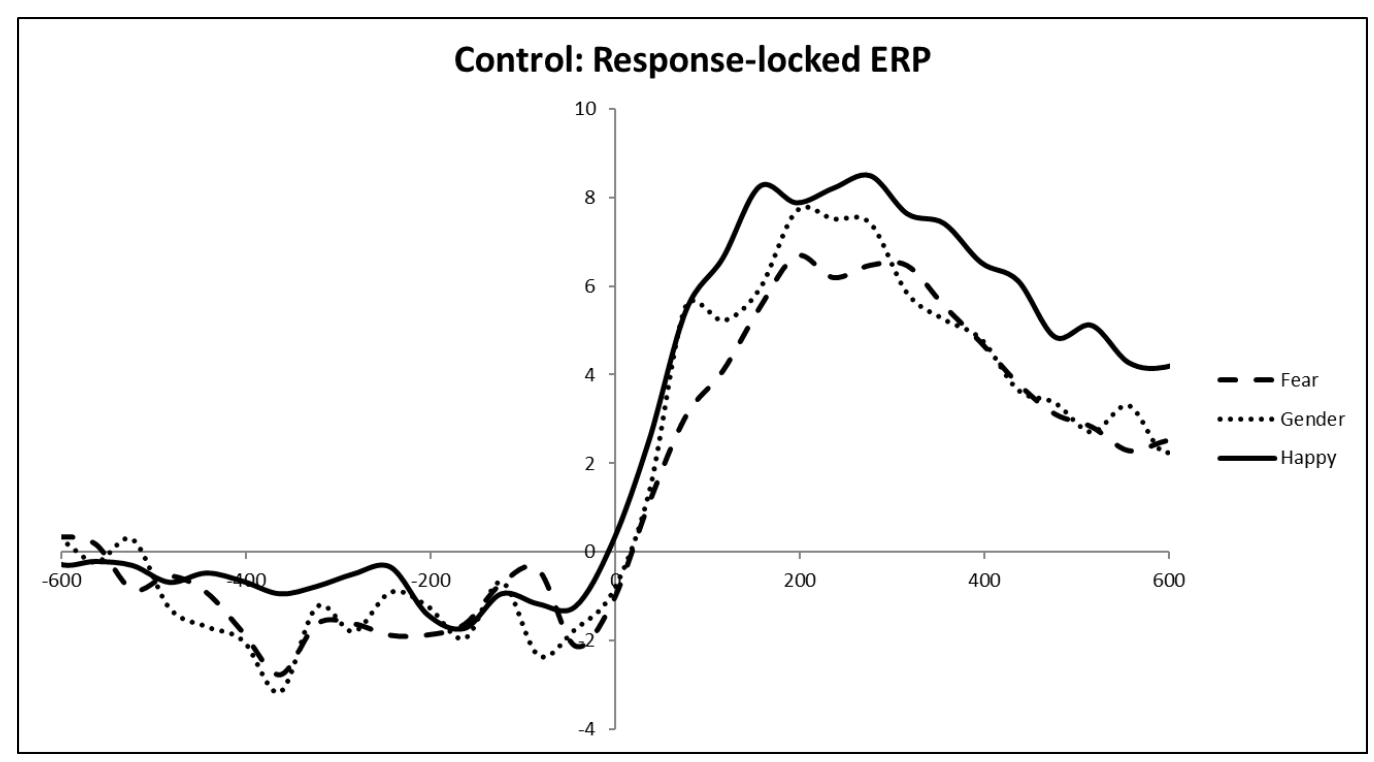


Figure 4. Post-error slowing interaction between group and emotion condition

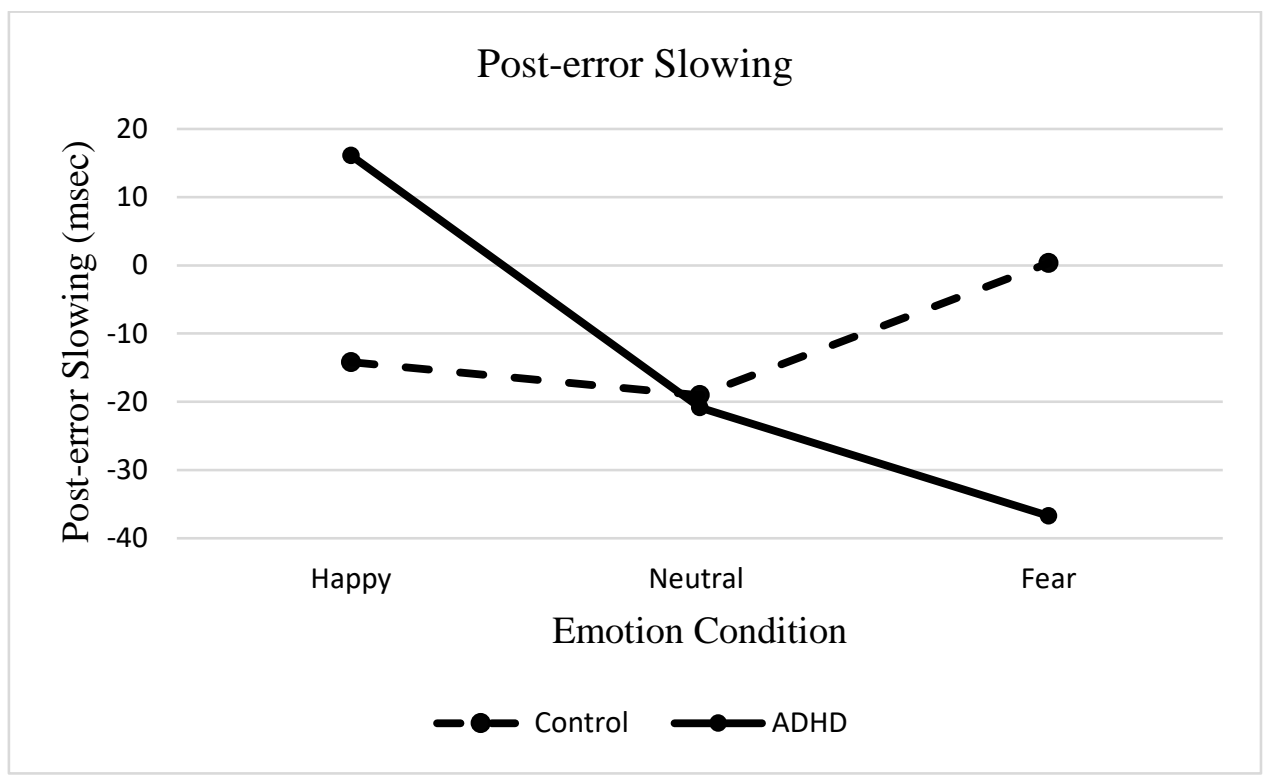

